\title{
Japanese version of the 42-item psychological well-being scale (PWBS-42): a validation study
}

\author{
Natsu Sasaki ${ }^{1}$, Kazuhiro Watanabe ${ }^{1}$, Kotaro Imamura', Daisuke Nishi', Mayumi Karasawa², Chiemi Kan³, \\ Carol Diane Ryff ${ }^{4}$ and Norito Kawakami ${ }^{1 *}$ (D)
}

\begin{abstract}
Background: The aim of this study was to examine the internal consistency, structural validity, and convergent/ known-group validity of the Japanese version of the 42-item Psychological Well-Being Scale (PWBS-42).

Methods: The PWBS-42 includes six 7-item subscales designed to measure the following dimensions of eudaimonic psychological well-being: 1) autonomy, 2) environmental mastery, 3) personal growth, 4) positive relations with others, 5) purpose in life, and 6) self-acceptance. A questionnaire was administered to 2102 community residents in Tokyo aged 30 or over as a part of the Midlife in Japan (MIDJA) survey, in 2008. The internal consistency reliability was tested using Cronbach's a. Structural validity was examined using exploratory factor analysis (EFA). Convergent validity was evaluated by calculating correlations of the Japanese PWBS-42 subscales with life satisfaction, negative affect, negative adjectives, positive affect, positive adjectives, self-esteem, and perceived stress scales.

Results: Data from 1027 respondents ( 505 males and 522 females) were analyzed (valid response rate $=56.2 \%$ ). Cronbach's a values ranged from 0.70 to 0.78 for five of the subscales, while that for purpose in life was lower (0.57). EFA yielded a five-factor structure: The first two factors consisted of negative and positive items mostly from the environmental mastery, purpose in life, and self-acceptance subscales. The third, fourth, and fifth factors consisted mostly of items from the positive relations with others, autonomy, and personal growth subscales, respectively. As hypothesized, the scores for life satisfaction, negative and positive affect/adjectives, self-esteem and perceived stress were significantly correlated with all subscales of the Japanese PWBS-42.

Conclusion: The subscales of the Japanese version of the PWBS-42 showed accep. levels of reliability and support for convergent validity in the Japanese population. The factor structure was slightly different from the theoretical 6factor model: items of three subscales (environmental mastery, purpose in life, and self-acceptance) loaded together on two factors. This finding may be interpreted in light of the interdependent self construal found in Japan in which these three components could be closely linked.
\end{abstract}

Keywords: Psychological well-being , PWB, Eudaimonic well-being, Positive mental health, Happiness, Meaning in life, Psychometric properties, Reliability, Validity, Flourishing structural invariance

\footnotetext{
*Correspondence: nkawakami@m.u-tokyo.ac.jp

${ }^{1}$ Department of Mental Health, Graduate School of Medicine, The University of Tokyo, 7-3-1, Hongo, Bunkyo-ku, Tokyo 113-0033, Japan

Full list of author information is available at the end of the article
}

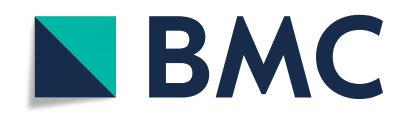

(c) The Author(s). 2020 Open Access This article is licensed under a Creative Commons Attribution 4.0 International License, which permits use, sharing, adaptation, distribution and reproduction in any medium or format, as long as you give appropriate credit to the original author(s) and the source, provide a link to the Creative Commons licence, and indicate if changes were made. The images or other third party material in this article are included in the article's Creative Commons licence, unless indicated otherwise in a credit line to the material. If material is not included in the article's Creative Commons licence and your intended use is not permitted by statutory regulation or exceeds the permitted use, you will need to obtain permission directly from the copyright holder. To view a copy of this licence, visit http://creativecommons.org/licenses/by/4.0/ The Creative Commons Public Domain Dedication waiver (http://creativecommons.org/publicdomain/zero/1.0/) applies to the data made available in this article, unless otherwise stated in a credit line to the data. 


\section{Background}

Eudaimonic well-being has been often discussed as purpose in life, meaning of life [1, 2], or as a concept relevant to self-determination theory $[3,4]$. One of the most famous models of eudaimonic well-being is the psychological well-being (PWB) model, developed by Carol D. Ryff [5]. PWB integrated psychological concepts such as self-actualization [6] and being a fully functioning person [7], distilling six key factors of well-being: autonomy, environmental mastery, personal growth, positive relations with others, purpose in life, and self-acceptance. The association of Ryff's PWB with various health outcomes has been well-documented [8]: overall longer survival [9], low risk of physical diseases such as stroke [10], myocardial infarction [11], and metabolic syndrome [12], and low risk of mental illness such as depression $[13,14]$, insomnia [15] and Alzheimer's disease [16]. In terms of biological functioning, higher levels of PWB associated with lower level of plasma cytokines $[17,18]$.

Since promoting PWB can contribute to people's personal growth, career development, and successful aging, measuring and promoting PWB among the Japanese population might be helpful for solving important issues in life at every stage. PWB has been frequently measured with Ryffs Psychological Well-Being Scales (PWBS) [19]. The reliability and validity of the PWBS has been established in more than 30 languages and across various cultures [20,21].

In Japan, three published articles have examined measurement of PWB in Japanese samples [22-24]. However, the measures the researchers used made it difficult to compare scores with those in other languages: two of them were not developed based on Ryff's original items of PWBS [22, 23], the other one [24] had 84 items which were rated dichotomously, with categories of "Yes" or "No". However, in cross-cultural cohort studies, it is more common for items to be rated on 6- or 7-point Likert scales. In addition, the measures used in these three studies have limitations in terms of balance between the burden on the participants of completing them and adequate depth of measurement, thus raising questions about the credibility of their assessments of the six well-being constructs. Although the length of the PWBS ranges from 18 [25] to 120 items [19], the ultra-short (18 items) version had psychometric problems of low internal consistency $(0.33<$ Cronbach's alpha $<0.56)$ [25], while acceptable criteria is regarded Cronbach's alpha $>0.7$. Moreover, the 84- and 120-items versions imposed an undue burden on the respondents $[8,19,26,27]$. The 42-item PWBS has been recently recognized as achieving the needed balance, and has been adopted in several studies including the Midlife in the United States (MIDUS) survey, a large cohort study in the United States $[8,28]$.

Hence, the purpose of this study is to examine the internal consistency, structural validity, and convergent/ known-group validity of the Japanese version of the 42item PWBS among the general population in Japan. In addition, we considered the interpretability of this measurement.

\section{Methods \\ Study design and participants}

This is a validation study for a self-report measure of well-being. The Midlife in Japan (MIDJA) study surveyed 2102 community residents aged $30-79$ years old living in the 23 wards of Tokyo city from April to September 2008 [29]. The responses were collected from 1027 adults ( 505 men and 522 women; age range $=30$ to 79 years). The overall response rate was $56.2 \%$. It was found that 275 participants were non-eligible (i.e., undelivered) due to having moved, unknown address, being absent during the time of the survey, illness, injury, being hospitalized or being deceased, which described in detail elsewhere (data available upon request). The primary objective of the MIDJA is to compare the Japanese sample (MIDJA) with the United States sample (MIDUS) to test hypotheses linking constructs of interdependence and independence to well-being and health. All respondents completed a self-administered questionnaire. The Research Ethics Committee of the Graduate School of Medicine in The University of Tokyo approved this study (no. 1691- [5]).

The study was reported according to the Consensusbased Standards for the Selection of Health Measurement Instruments (COSMIN) guideline, which is used to improve the quality of efforts to develop health-related selfreport measurement instruments [30]. Each finding for properties of measurement in this study was assessed by the COSMIN Risk of Bias checklist (Additional file 1).

\section{Measurements}

\section{Psychological well-being scale (PWBS)}

With permission from Professor Ryff, the developer of the PWBS, we translated the 42 items of the PWBS into Japanese. The PWBS [19] originally comprised six subscales with seven items each to measure the six factors: 1) autonomy (e.g., I am not afraid to voice my opinions, even when they are in opposition to the opinions of most people.); 2) environmental mastery (e.g., In general, I feel I am in charge of the situation in which I live.); 3) personal growth(e.g., I think it is important to have new experiences that challenge how I think about myself and the world.); 4) positive relations with others(e.g., Most people see me as loving and affectionate.); 5) purpose in life(e.g., I have a sense of direction and purpose in life.); and 6) self-acceptance(e.g., In general, I feel confident and positive about myself.). Response categories for these items are on a seven-point scale: ranging from 1 ("Strongly disagree") to 7 ("Strongly agree"). The scores 
for six subscales were calculated as averages; higher scores mean greater psychological well-being.

The authors complied with the International Society for Pharmacoeconomics and Outcomes Research (ISPOR) taskforce guidelines [31], which constitute the standard procedure for translation and adaptation of self-reporting scales in other languages. The forward (from English to Japanese) translation of the PWBS 42 items was prepared by Prof. Karasawa, who is a research member of the MIDJA, with considerable knowledge about the theoretical background of PWB. The forward translation was reviewed and revised by colleagues at The University of Tokyo to confirm its relevance and meaningfulness. After these consultations with researchers, the slightly amended version was back-translated into English by an independent translator who was blind to the original English version and reviewed by research members of MIDJA. The original developer, Prof. Ryff, reviewed this back translation and further corrections were made based on her suggestions.

\section{Life satisfaction (6 items)}

Life satisfaction was measured by the newly developed six items scale for the MIDUS/MIDJA (e.g., "How would you rate your life overall these days"). Assessing overall, work, health, family (the mean of ratings for relationship with spouse/partner and relationship with children) and financial satisfaction each question asked participants about their satisfaction with their life on current days on a scale ranging from 0 (the worst possible) to 10 (the best possible). The overall score was constructed by calculating the mean of the items. The scale was computed for cases that had valid values for at least one item on the scale. Higher scores reflect higher levels of overall life satisfaction, meaning high hedonic well-being. In this study sample, the internal consistency reliability of the scale (Cronbach's alpha) was 0.75 [32].

\section{Positive and negative affect/adjective}

Positive and negative adjectives were measured by four and five items of each (e.g., enthusiastic, attentive, proud, active, afraid, jittery, irritable, ashamed, upset) from The Positive and Negative Affect Schedule (PANAS) [33], which is a widely used mood measurement and regarded as one of the indicators of hedonic well-being. In addition, positive affect was each measured by six items (i.e., cheerful, in good spirits, extremely happy, calm and peaceful, satisfied, full of life), because such low arousal positive feeling has been reported to have stronger link with health among Japanese people, other than high arousal feelings, measured by PANAS [34]. The negative affect was also measured by an original six-item scale consisting one item on "nervousness" from PANAS and items from other sources (i.e., so sad nothing could cheer you up, restless or fidgety, hopeless, that everything was an effort, worthless). Additional items both in positive and negative affect were retrieved from the previous study [35]. This study used the past 30 days as the time frame (i.e.., During the past 30 days, how much of the time did you feel...). All items were rated on a 5-point Likert-type scale, ranging from 1 "None of the time" to 5 "All of the time". Scales were constructed by calculating the mean across each set of items. The scales were computed for cases that had valid values for at least one item on the scale. In this study sample, the internal consistency reliability (Cronbach's alpha) of the scales for 1) positive affect, 2) positive adjective, 3) negative affect, 4) negative adjective was 1) $0.93,2) 0.80,3$ ) 0.86 and 4) 0.82 respectively [32]. We used these four sub-scales as a measurement of affect to keep their independent construction. Positive affect and adjective in this questionnaire is well validated elsewhere [34].

\section{Self-esteem (7 items)}

Self-esteem was measured with a scale consisting of seven items taken from Rosenberg's self-esteem scales [36] (e.g., "I am able to do things as well as most people"). A response to each item was scored on a seven-point scale ranging from 1 (agree strongly) to 7 (disagree strongly). In this study sample, Cronbach's alpha for men and women was 0.63 and 0.69 [37], respectively.

\section{Perceived stress (10 items)}

Perceived stress was measured with the Perceived Stress Scale (PSS) [38, 39], which asked 10 items about their recognized stressors on these 30 days (e.g., "felt that you were unable to control the important things in your life?"). Each item was rated on five-point scale ranging from 1 (Never) to 5 (Very often). In this study sample, Cronbach's alpha was 0.76 [37].

\section{Demographic variables}

A questionnaire was administered to assess demographic variables, including gender (male or female), age, education status (Junior high school, some high school, high school, vocational school, 2 year college, some college, 4 or 6 year college or over graduate school), marital status (married, divorced/widowed or single) and work status (Have a paid job or Do not have a paid job).

\section{Statistical analysis}

Statistical significance was defined as $p<0.05$. All the statistical analyses were performed using SPSS 26.0, Japanese version (SPSS Inc., Chicago, IL).

Before conducting the analyses, the scores of some items were reversed as recommended in Ryff's original PWBS [19]. For an item with a missing value, the mean value of the completed items was imputed. The scales were computed for cases that had valid values for at 
least one item in each domain following the documentation of "Scales and Constructed Variables in MIDJA" guide [32].

To assess the internal consistency of the Japanese PWBS, Cronbach's alpha $(\alpha)$ coefficients were calculated for each of the six subscales. To assess structural validity, confirmatory factor analysis (CFA) was conducted to test the goodness of fit for the existing structure of psychological well-being, and an exploratory factor analysis (EFA) was conducted in case the CFA showed a poor fit. Based on previous research, we hypothesized a six-factor structure. In addition, we extracted factors with eigenvalues of more than 1.0, following the Kaiser-Guttman "Eigenvalues greater than one" criterion [40-42], using robust maximum likelihood estimation. In the two EFAs, the promax rotation method was adopted to calculate factor loadings for the items. As a hypothesis test for convergent validity, Pearson's correlation coefficients (rs) were calculated between each score of the PWBS and life satisfaction, positive and negative affect/adjectives, and perceived stress, which was considered to have moderate-to-strong associations with psychological wellbeing. Positive and moderate correlations would be expected with life satisfaction, positive affect/adjectives, and self-esteem. Negative and moderate correlations would be expected with negative affect/adjectives and perceived stress. Although these measures consisted of distinct components of well-being, prior research revealed relations among evaluative, hedonic and eudaimonic well-being [43]. In addition, a one-way factorial ANOVA was performed as another hypothesis test for known-group validity to compare the scores of the six subscales of the Japanese PWBS when stratified based on demographic and occupational variables (sex, age, marital status and working status). For these demographic and occupational variables, we observed significant differences from the PWB scores in previous studies (e.g., high personal growth and purpose in life in the elderly, high positive relationship with others in women) [25].

\section{Results}

\section{Characteristics of participants}

We received responses from 1027 members (56.2\%) of the target sample. The proportion of males was $49.2 \%$, and average age was 54.4 years old. The demographic characteristics of the participants are shown in Table 1. Many of the participants were married (69.1\%) and had a paid job (71.6\%).

\section{Internal consistency}

Table 2 shows mean scores and Cronbach's alphas $(\alpha)$ for the PWBS-42 subscales. The Cronbach's $\alpha$ coefficients ranged from 0.70 to 0.78 , except for that for Purpose in life $(\alpha=0.57)$.
Table 1 Participant characteristics. $(N=1027)$

\begin{tabular}{ll}
\hline & $\mathrm{N}(\%)$ \\
\hline Sex(male) & $505(49.2)$ \\
Age mean, SD & $54.4,14.1$ \\
$30-39$ & $200(19.5)$ \\
$40-49$ & $215(20.9)$ \\
$50-59$ & $206(20.1)$ \\
$60-69$ & $200(19.5)$ \\
$70 \leqq$ & $206(20.1)$ \\
Marital status & \\
Married & $710(69.1)$ \\
Divorsed/Widowed & $149(14.5)$ \\
Single & $166(16.2)$ \\
Missing & $2(0.2)$ \\
Working status & \\
Have a paid job & $735(71.6)$ \\
No paid job & $289(28.1)$ \\
Missing & $3(0.3)$ \\
Education & \\
Junior high school & \\
Some high school & $97(9.4)$ \\
High school & $33(3.2)$ \\
Vocational school & $306(29.8)$ \\
Somear college college & $139(13.5)$ \\
Over 6 year college & $26(2.5)$ \\
Missing & $300(29.2)$ \\
\hline
\end{tabular}

\section{Factor structure of PWBS-42 in Japanese}

The results of CFA are shown in Table 3. The original hypothesized six-factor model demonstrated poor fit $\left(\mathrm{X}^{2}\right.$ $[804]=6651, \mathrm{CFI}=0.638, \mathrm{TLI}=0.594, \mathrm{RMSEA}=0.084)$. The results of EFA assuming a six factor structure indicated that the 42 items loaded on five of the six factors

Table 2 Average, standard deviation (SD) and reliability among Japanese population for the PWB domains. $(N=1027)$

\begin{tabular}{llll}
\hline PWB a domains (Possible range: 7-49) & Mean & SD & Cronbach's a \\
\hline Autonomy & 30.6 & 5.3 & 0.70 \\
Environmental Mastery & 31.7 & 5.4 & 0.74 \\
Personal growth & 33.8 & 5.6 & 0.75 \\
Positive relationship with others & 33.5 & 5.7 & 0.76 \\
Purpose in life & 31.8 & 5.0 & 0.57 \\
Self-acceptance & 30.8 & 5.7 & 0.78 \\
\hline
\end{tabular}

Participants who had missing data were analyzed according to the algorithm described in the Method section

$S D$ standard deviation

a PWB Psychological well-being 
Table 3 Factor loadings of PWB 42 items and model fit in confirmatory factor analyses

\begin{tabular}{ll}
\hline Model fit & 6-factor \\
\hline X2(df) & $6651(804)$ \\
CFI & 0.638 \\
TLI & 0.594 \\
RMSEA $(95 \% \mathrm{Cl})$ & $0.084(0.082-0.086)$
\end{tabular}

Factor loadings ${ }^{a}$

Autonomy

A1 $\quad 0.676$

A2 $\quad 0.448$

A3 $\quad 0.402$

A4 $\quad 0.402$

0.402

A5 $\quad 0.660$

A6 $\quad 0.529$

A7 $\quad 0.351$

Positive relation with others

R1 $\quad 0.477$

R2 0.531

R3 $\quad 0.660$

R4 $\quad 0.563$

R5 $\quad 0.405$

R6 $\quad 0.681$

R7 $\quad 0.382$

${ }^{a}$ All items showed significant factor loading

and no item loaded on the sixth factor (data available upon request). Then, we tried conducting another EFA that hypothesized a 5-factor structure with the promax rotation method, which allows factors to correlate with each other, using a robust maximum likelihood estimation. Table 4 shows that the EFA then successfully yielded five factors. The first two factors consisted of negative and positive items mostly from environmental mastery, purpose in life, and self-acceptance. The third, fourth, and fifth factors consisted mostly of items for positive relations with others, autonomy, and personal growth, respectively. EFA according to the Kaiser-Guttman's criterion indicated an eight-factor structure (data available upon request). Authors did not adopt the model because it was difficult to interpret the meaningfulness of the eight factors and there were few items loaded on the subordinate factors.

Table 5 shows correlations between the scores of the theoretical subscales that originally assumed on the PWBS-42 and scores of the five factors extracted from the previous EFA. Each factor score was sum of items. There were significant and exclusive correlation between the five factors and the six subscales of the PWBS-42; Factor 2 and Self-acceptance, Factor 3 and Positive relationship with others, Factor 4 and Autonomy, and Factor 5 and Personal growth. Factor 1 showed moderate association with all theoretical six subscales.

\section{Hypothesis testing}

\section{Convergent validity}

Table 6 shows correlations between the scores of the six subscales of the PWBS-42 and life satisfaction, positive and negative affect/adjectives, self-esteem, and perceived stress. Although the EFA showed a five-factor structure, we presented average scores and correlations of the original six subscales because it was planned as part of validation and for keeping the international comparability. In addition, we cannot conclude that the original six-factor structure and the observed five-factor structure are definitely different as shown in Table 5. All scales were significantly correlated with all domains of PWB. Scales related to negative feelings (i.e., negative affect, adjective, PSS) were correlated negatively with all domains $(-0.55<\mathrm{r}<-0.13)$. Positive scales (i.e., life satisfaction, positive affect, adjectives, self-esteem) were correlated positively with all domains $(0.24<\mathrm{r}<0.71)$.

\section{Known-group validity}

Table 7 shows the results of ANOVAs to examine the differences between each category for the demographic characteristics (sex, age, marital and working status). One-way ANOVA revealed some significant differences among variables (e.g., high autonomy in males, high personal growth in workers).

\section{Discussion}

The results showed an acceptable level of internal consistency reliability of the newly translated Japanese version of the PWBS-42, although careful consideration of the results was need given that factor analysis showed a five-factor structure. Cronbach's alpha coefficient was lower for the scales for purpose in life, possibly because one item of "Done all there is to do in life" (the item P7) behaved differently than the other items. The results of CFA did not show a good model fit for the previously proposed six-factor structure model. The correlation analysis showed theoretically high correlation with other scales, supporting convergent validity. The ANOVA confirmed the hypothesized differences according to demographic characteristics between known independent groups (population in U.S. [25] vs. Japan), suggesting good known-group validity, as described in detail below.

We found that the original six-factor model [5] did not fit well with the present data in the CFA. Rather, the EFA suggested a five-factor structure. The first two factors 
Table 4 Exploratory factor analysis assuming a five-factor structure by using maximum likelihood with Promax rotation

\begin{tabular}{|c|c|c|c|c|c|c|c|}
\hline \multirow[t]{2}{*}{ - } & \multirow[t]{2}{*}{ Reverse } & \multirow[t]{2}{*}{ Item } & \multicolumn{5}{|c|}{ Factor loading score } \\
\hline & & & 1 & 2 & 3 & 4 & 5 \\
\hline E6 & $\mathrm{R}$ & Difficult arranging life in satisfying way & 0.682 & 0.170 & -0.019 & 0.059 & -0.090 \\
\hline E2 & $\mathrm{R}$ & Demands of everyday life often get me down & 0.633 & 0.031 & -0.101 & 0.008 & -0.107 \\
\hline E5 & R & Overwhelmed by my responsibilities & 0.628 & 0.023 & -0.134 & 0.135 & -0.065 \\
\hline A6 & $\mathrm{R}$ & Worry about what others think of me & 0.600 & 0.070 & -0.147 & 0.236 & -0.175 \\
\hline S5 & $\mathrm{R}$ & Disappointed about achievements in life & 0.592 & 0.165 & 0.101 & -0.118 & 0.058 \\
\hline P4 & $\mathrm{R}$ & Daily activities seem trivial \& unimportant & 0.531 & 0.057 & -0.015 & -0.089 & 0.153 \\
\hline R2 & $\mathrm{R}$ & Maintaining close relationships difficult & 0.531 & -0.109 & 0.212 & 0.073 & -0.043 \\
\hline S6 & $\mathrm{R}$ & Self attitude not as positive as others & 0.520 & 0.222 & $0-.029$ & -0.127 & 0.048 \\
\hline S3 & R & Others have gotten more out of life than me & 0.507 & -0.032 & -0.082 & 0.018 & 0.001 \\
\hline R3 & R & Few close friends to share concerns with & 0.501 & -0.076 & 0.339 & -0.088 & 0.037 \\
\hline E3 & $\mathrm{R}$ & Don't fit in with people and community & 0.492 & -0.074 & 0.264 & -0.045 & -0.012 \\
\hline G3 & R & Haven't improved as person over years & 0.488 & 0.285 & -0.013 & -0.140 & 0.143 \\
\hline R6 & $\mathrm{R}$ & No experience with warm \& trusting relations & 0.487 & -0.135 & 0.300 & -0.094 & 0.231 \\
\hline G6 & R & Gave up trying to make improvements long ago & 0.461 & 0.065 & 0.025 & -0.036 & 0.397 \\
\hline P3 & $\mathrm{R}$ & No good sense of what I'm trying to accomplish & 0.458 & 0.401 & -0.160 & -0.139 & 0.150 \\
\hline G7 & $\mathrm{R}$ & Do not enjoy situations requiring a change in my ways & 0.441 & -0.129 & -0.005 & 0.097 & 0.135 \\
\hline A3 & $\mathrm{R}$ & Influenced by people with strong opinions & 0.432 & -0.158 & -0.115 & 0.305 & -0.079 \\
\hline S7 & & Feel good when compare myself to friends & 0.071 & 0.703 & 0.174 & -0.021 & -0.184 \\
\hline P7 & $\mathrm{R}$ & Done all there is to do in life & 0.059 & -0.684 & 0.067 & 0.047 & 0.350 \\
\hline S2 & & Feel positive/confident about self & 0.081 & 0.595 & -0.085 & 0.209 & 0.146 \\
\hline G4 & & Developed a lot as person over time & 0.052 & 0.560 & 0.183 & -0.003 & 0.036 \\
\hline P5 & & Enjoy making plans for future and making it real & -0.091 & 0.520 & -.044 & -0.002 & 0.379 \\
\hline P2 & & Have sense of direction/purpose in life & -0.100 & 0.509 & -0.142 & 0.115 & 0.483 \\
\hline S1 & & Pleased with how life turned out & 0.103 & 0.480 & 0.196 & -0.082 & -0.057 \\
\hline E4 & & Good at managing daily responsibilities & 0.051 & 0.465 & 0.131 & 0.241 & -0.113 \\
\hline A4 & & Confidence in my opinions even if others are contrary & -0.221 & 0.427 & -0.018 & 0.371 & 0.083 \\
\hline S4 & & Like most aspects of my personality & 0.147 & 0.424 & 0.283 & 0.075 & -0.136 \\
\hline E7 & & Able to build lifestyle to my liking & 0.148 & 0.424 & 0.176 & 0.100 & $0-.105$ \\
\hline P6 & & Some wander aimlessly but not me & -0.040 & 0.369 & 0.113 & -0.011 & 0.082 \\
\hline R7 & & I can trust friends \& they can trust me & 0.003 & 0.144 & 0.679 & 0.073 & -0.092 \\
\hline R4 & & Enjoy conversations with family \& friends & -0.004 & 0.132 & 0.508 & 0.037 & 0.080 \\
\hline R1 & & Most see me as loving/affectionate & -0.094 & 0.191 & 0.495 & 0.051 & 0.000 \\
\hline R5 & & Others describe me as giving/share time & -0.116 & 0.292 & 0.386 & 0.019 & -0.025 \\
\hline A1 & & Not afraid to voice opposing opinions & 0.095 & -0.020 & 0.037 & 0.694 & 0.103 \\
\hline A5 & $\mathrm{R}$ & Difficulty voicing opinion on controversial issues & 0.461 & -0.147 & -0.068 & 0.498 & 0.138 \\
\hline E1 & & In charge of situation in which I live & 0.110 & 0.142 & 0.207 & 0.450 & 0.070 \\
\hline A2 & & Decisions not influenced by others' actions & -0.019 & 0.286 & $0-.007$ & 0.389 & -0.088 \\
\hline A7 & & Judge self by what I think is important & -0.090 & 0.070 & 0.102 & 0.379 & 0.038 \\
\hline G5 & & Life process of learning/changing/growth & -0.133 & 0.217 & 0.214 & 0.047 & 0.460 \\
\hline G2 & & Important to experience the challenge how to think about yourself and the world & -0.233 & -0.037 & 0.199 & 0.236 & 0.447 \\
\hline G1 & $\mathrm{R}$ & Not interested in activities to expand horizons & 0.296 & -0.231 & 0.043 & 0.087 & 0.446 \\
\hline P1 & R & Live life day to day, don't think about future & 0.202 & -0.132 & -0.185 & -0.075 & 0.432 \\
\hline
\end{tabular}


Table 5 Pearson's correlation coefficients ${ }^{a}$ between the each score of PWB and each score of factors which extracted from factorial analysis

\begin{tabular}{|c|c|c|c|c|c|c|c|c|c|}
\hline \multirow{2}{*}{$\begin{array}{l}\text { Variables } \\
\text { [number of item] } \\
\text { (minimum- } \\
\text { maximum) }\end{array}$} & \multirow[t]{2}{*}{$\mathrm{N}$} & \multirow[t]{2}{*}{ Mean } & \multirow[t]{2}{*}{ SD } & \multicolumn{6}{|c|}{ Psychological well-being (PWB) domains } \\
\hline & & & & Autonomy & $\begin{array}{l}\text { Environmental } \\
\text { Mastery }\end{array}$ & $\begin{array}{l}\text { Personal } \\
\text { growth }\end{array}$ & $\begin{array}{l}\text { Positive relationship with } \\
\text { others }\end{array}$ & $\begin{array}{l}\text { Purpose in } \\
\text { life }\end{array}$ & $\begin{array}{l}\text { Self- } \\
\text { acceptance }\end{array}$ \\
\hline $\begin{array}{l}\text { Factor } 1 \text { [17] (33- } \\
\text { 119) }\end{array}$ & 1024 & 77.65 & 14.0 & $0.508^{* *}$ & $0.825^{* *}$ & $0.734^{* *}$ & $0.718^{* *}$ & $0.629^{* *}$ & $0.724^{* *}$ \\
\hline $\begin{array}{l}\text { Factor } 2 \text { [12] (17- } \\
\text { 78) }\end{array}$ & 1021 & 53.49 & 8.4 & $0.499^{* *}$ & $0.638^{* *}$ & $0.646^{* *}$ & $0.572^{* *}$ & $0.667^{* *}$ & $0.811^{* *}$ \\
\hline Factor 3 [4] (4-28) & 1025 & 18.77 & 3.3 & $0.256^{* *}$ & $0.456^{* *}$ & $0.498^{* *}$ & $0.853^{* *}$ & $0.402^{* *}$ & $0.524^{* *}$ \\
\hline $\begin{array}{l}\text { Factor } 4[5](10- \\
35)\end{array}$ & 1021 & 22.67 & 4.1 & $0.896^{* *}$ & $0.556^{* *}$ & $0.446^{* *}$ & $0.357^{* *}$ & $0.339^{* *}$ & $0.442^{* *}$ \\
\hline Factor 5 [4] (8-28) & 1024 & 19.65 & 3.3 & $0.267^{* *}$ & $0.338^{* *}$ & $0.771^{* *}$ & $0.444^{* *}$ & $0.646^{* *}$ & $0.349^{* *}$ \\
\hline
\end{tabular}

SD standard deviation

a Participants who had missing data were excluded from each analysis

** $p<0.01, * 0.05$

consisted of negatively and positively worded items mostly from environmental mastery, purpose in life, and selfacceptance, as well as some items from the other subscales. The result for the correlation analysis also showed that Factor 1 and 2 was highly related $(r>0.8)$ with environmental mastery and self-acceptance. Factor 1 had high correlation $(r>0.7)$ with most of PWB subscales instead of autonomy and purpose in life. The highest correlation among them was with environmental mastery, identifying the EFA result that the top three items of high loading score in Factor 1 were all about environmental mastery. The correlation of Factor 2 and self-acceptance may possibly be due to relatively high loading scores of S7 and S2. Although purpose in life did not show high correlation with any factors, it might be due to its low internal consistency. We discuss two aspects of the possible reasons the factor analysis yielded these results.
First, it seems that respondents in this sample of Japanese community residents perceived three constructs measured by these subscales (i.e., environmental mastery, purpose in life, and self-acceptance) quite similar to each other. The findings contract to previous observations in Western countries that these constructs are separate in factor analyses [25, 44]. Among Eastern cultures, the self is viewed as interdependent, rather than independent as it is in Western culture. Experiencing interdependence entails seeing oneself as part of a network of social relationships and recognizing that one's behavior is determined by the others' perspectives or expectations. The fundamental connectedness of the human community with the surrounding context "self-inrelation-to-other" is focal in individual experiences in Eastern cultures [45]. With the interdependent self, wellbeing is realized in one's relationship with surrounding

Table 6 Pearson's correlation coefficients ${ }^{a}$ between each subscale on the PWB and Life Satisfaction, Negative Affect, Negative Adjectives, Positive Affect, Positive Adjectives, Self-esteem, Percieved Stress Scale (PSS) among Japanese population

\begin{tabular}{|c|c|c|c|c|c|c|c|c|c|}
\hline \multirow{2}{*}{$\begin{array}{l}\text { Variables (possible } \\
\text { range) }\end{array}$} & \multirow[t]{2}{*}{$\mathrm{N}$} & \multirow[t]{2}{*}{ Mean } & \multirow[t]{2}{*}{ SD } & \multicolumn{6}{|c|}{ Psychological well-being (PWB) domains } \\
\hline & & & & Autonomy & $\begin{array}{l}\text { Environmental } \\
\text { Mastery }\end{array}$ & $\begin{array}{l}\text { Personal } \\
\text { growth }\end{array}$ & $\begin{array}{l}\text { Positive relationship with } \\
\text { others }\end{array}$ & $\begin{array}{l}\text { Purpose in } \\
\text { life }\end{array}$ & $\begin{array}{l}\text { Self- } \\
\text { acceptance }\end{array}$ \\
\hline Life Satisfaction (0-10) & 1027 & 6.10 & 1.58 & $0.235^{* *}$ & $0.528^{* *}$ & $0.382^{* *}$ & $0.412^{* *}$ & $0.357^{* *}$ & $0.562^{* *}$ \\
\hline \multicolumn{10}{|c|}{ Positive and negative affect } \\
\hline $\begin{array}{l}\text { Negative Affect (1- } \\
\text { 5) }\end{array}$ & 1024 & 1.69 & 0.65 & $-0.212^{* *}$ & $-0.419^{* *}$ & $-0.218^{* *}$ & $-0.293^{* *}$ & $-0.207^{* *}$ & $-0.408^{* *}$ \\
\hline $\begin{array}{l}\text { Negative Adjectives } \\
(1-5)\end{array}$ & 1021 & 1.89 & 0.66 & $-0.245^{* *}$ & $-0.354^{* *}$ & $-0.126^{* *}$ & $-0.205^{* *}$ & $-0.147^{* *}$ & $-0.285^{* *}$ \\
\hline Positive Affect [1-3] & 1022 & 3.24 & 0.76 & $0.220^{* *}$ & $0.446^{* *}$ & $0.380^{* *}$ & $0.464^{* *}$ & $0.302^{* *}$ & $0.509^{* *}$ \\
\hline $\begin{array}{l}\text { Positive Adjectives } \\
(1-5)\end{array}$ & 1020 & 3.07 & 0.76 & $0.344^{* *}$ & $0.427^{* *}$ & $0.421^{* *}$ & $0.394^{* *}$ & $0.350^{* *}$ & $0.445^{* *}$ \\
\hline Self-esteem (7-49) & 1021 & 31.02 & 5.58 & $0.463^{* *}$ & $0.646^{* *}$ & $0.509^{* *}$ & $0.470^{* *}$ & $0.433^{* *}$ & $0.714^{* *}$ \\
\hline PSS (10-50) & 1008 & 26.11 & 5.77 & $-0.306^{* *}$ & $-0.554^{* *}$ & $-0.298^{* *}$ & $-0.370^{* *}$ & $-0.295^{* *}$ & $-0.511^{* *}$ \\
\hline
\end{tabular}

SD standard deviation

a Participants who had missing data were excluded from each analysis

${ }^{* *} p<0.01,{ }^{*}<0.05$ 
Table 7 Comparison with the participant characteristics for four scales of PWBS-42 in Japanese (One-way ANOVA) (N=1027)

\begin{tabular}{|c|c|c|c|c|c|c|c|c|c|}
\hline \multirow[b]{2}{*}{ Category (N) } & \multicolumn{3}{|l|}{ Autonomy } & \multicolumn{3}{|c|}{ Environmental Mastery } & \multicolumn{3}{|c|}{ Personal growth } \\
\hline & Mean (SD) & $\mathrm{F}$ & $p$ & Mean (SD) & $\mathrm{F}$ & $\mathrm{p}$ & Mean (SD) & $\mathrm{F}$ & $\mathrm{p}$ \\
\hline Sex & & 12.86 & $<0.001$ & & 3.15 & 0.076 & & 6.52 & 0.011 \\
\hline Male (505) & $31.2(5.3)$ & & & $31.4(5.8)$ & & & $33.3(5.6)$ & & \\
\hline Female (522) & $30.1(5.2)$ & & & $32.0(5.0)$ & & & $34.2(5.6)$ & & \\
\hline Age & & 4.02 & 0.003 & & 4.03 & 0.003 & & 6.66 & $<0.001$ \\
\hline 30-39 (200) & $29.5(5.9)$ & & & $30.6(5.3)$ & & & $34.5(5.9)$ & & \\
\hline 40-49 (215) & $30.4(5.7)$ & & & $31.5(5.4)$ & & & $34.7(5.5)$ & & \\
\hline 50-59 (206) & $30.8(5.4)$ & & & $32.5(6.0)$ & & & $34.3(5.8)$ & & \\
\hline 60-69 (200) & $31.2(4.9)$ & & & $32.2(5.5)$ & & & $33.1(5.6)$ & & \\
\hline $70 \leqq(206)$ & $31.3(4.4)$ & & & $31.6(4.5)$ & & & $32.4(5.0)$ & & \\
\hline Marital status & & 0.53 & 0.586 & & 11.25 & $<0.001$ & & 8.26 & $<0.001$ \\
\hline Married (710) & $30.7(5.3)$ & & & $32.2(5.4)$ & & & $34.2(5.5)$ & & \\
\hline Divorced/Widowed (149) & $30.6(4.7)$ & & & $31.3(5.3)$ & & & $33.1(5.8)$ & & \\
\hline Single (166) & $30.2(6.0)$ & & & $30.0(5.3)$ & & & $32.4(5.9)$ & & \\
\hline Working status & & 1.87 & 0.171 & & 0.20 & 0.656 & & 15.39 & $<0.001$ \\
\hline Have a paid job (735) & $30.8(5.5)$ & & & $31.8(5.6)$ & & & $34.2(5.8)$ & & \\
\hline \multirow[t]{2}{*}{ No paid job (289) } & $30.3(4.9)$ & & & $31.6(5.0)$ & & & $32.7(5.1)$ & & \\
\hline & \multicolumn{3}{|c|}{ Positive relationship with others } & \multicolumn{3}{|c|}{ Purpose in life } & \multicolumn{3}{|c|}{ Self-acceptance } \\
\hline Category (N) & Mean (SD) & F & $\mathrm{p}$ & Mean (SD) & $\mathrm{F}$ & $\mathrm{p}$ & Mean (SD) & $\mathrm{F}$ & $P$ \\
\hline Sex & & 30.12 & $<0.001$ & & 0.75 & 0.387 & & 2.87 & 0.091 \\
\hline Male (505) & $32.5(6.0)$ & & & $31.6(5.2)$ & & & $30.5(5.7)$ & & \\
\hline Female (522) & $34.5(5.3)$ & & & $31.9(4.8)$ & & & $31.1(5.7)$ & & \\
\hline Age & & 0.912 & 0.456 & & 2.66 & 0.032 & & 1.47 & 0.209 \\
\hline 30-39 (200) & $33.4(6.1)$ & & & $32.2(5.5)$ & & & $30.3(6.3)$ & & \\
\hline 40-49 (215) & $33.5(6.2)$ & & & $31.9(5.1)$ & & & $31.1(6.2)$ & & \\
\hline $50-59(206)$ & $34.1(5.6)$ & & & $32.4(5.1)$ & & & $31.5(5.9)$ & & \\
\hline $60-69(200)$ & $33.5(5.3)$ & & & $31.3(5.1)$ & & & $30.4(5.1)$ & & \\
\hline $70 \leqq(206)$ & $33.0(5.4)$ & & & $31.1(4.3)$ & & & $30.7(4.7)$ & & \\
\hline Marital status & & 15.56 & $<0.001$ & & 12.61 & $<0.001$ & & 21.28 & $<0.001$ \\
\hline Married (710) & $34.1(5.4)$ & & & $32.2(5.0)$ & & & $31.5(5.5)$ & & \\
\hline Divorced/Widowed (149) & $32.9(5.5)$ & & & $31.5(5.0)$ & & & $29.9(6.2)$ & & \\
\hline Single(166) & $31.5(6.6)$ & & & $30.1(4.9)$ & & & 28.6(5.4) & & \\
\hline Working status & & 0.11 & 0.745 & & 8.85 & 0.003 & & 1.06 & 0.304 \\
\hline Have a paid job (735) & $33.6(5.9)$ & & & $32.1(5.1)$ & & & $31.0(5.9)$ & & \\
\hline No paid job (289) & $33.4(5.2)$ & & & $31.0(4.8)$ & & & $30.5(5.1)$ & & \\
\hline
\end{tabular}

SD standard deviation

realities, both social and non-social [46]. Thus, environmental mastery, being able to take adaptive and situationally bound actions in the social context, might be closely linked with self-acceptance in a country with an Eastern culture, such as Japan. In addition, also in line with the construal of interdependent cultures, the self becomes most meaningful (i.e., high purpose in life) when it is cast in the appropriate social settings [45]. Japanese people are motivated to find a way to fit in with relevant surroundings by fulfilling obligations to become part of various interpersonal relationships [45]. Purpose in life may be also closely related to environmental mastery and self-acceptance in Japanese culture. In Japanese culture, these three components of PWB may come together and be hard to distinguish.

Second, negative and positive items were divided into two different factors in this sample. The same pattern was shown in a previous study with a UK cohort [47]. 
There are two reasons that the PWB scale includes negative/positive distinction of wordings; (1) assessing constructs with items framed positively and negatively is needed as a check on acquiescence response bias (i.e., the tendency to agree with everything, perhaps due to not even reading the items); (2) items for the PWB scales were generated based on the theoretical definitions of each construct which included a definition of both high and low scorers for each dimension (i.e., items intended to capture both having and not having the various aspects of well-being) [48]. The use of both negatively and positively worded items is expected to increase the validity of what is meant by having a high score on any dimension of well-being because to get a high score, a person has to both agree with positively worded items and disagree with negatively worded items. The pattern that negatively and positively worded items load on different factors in a factor analysis has been observed for several mental health and psychological well-being scales in Japan [49-52], which is interpreted as differential response styles to negatively and positively worded items in the Japanese population. Thus, the finding for the two factors may not necessarily imply that there are two distinct unique constructs of psychological well-being in the Japanese population. The two factors observed in the exploratory factor analysis may be just due to a reporting style, but still reflect the same construct.

Most items for the remaining three subscales (i.e., positive relations with others, autonomy, and personal growth) loaded on separate factors that seem to reflect the corresponding dimensions of the PWB. The results for the correlation analysis also suggested that Factor 3, 4, and 5 had high to moderate correlation with positive relationship with others, autonomy, and personal growth respectively. The observed five factor-structure in this study seem compatible with the original six-factor model [19] to some extent, while the three subscales (i.e, environmental mastery, purpose in life, and self-acceptance) were collapsed to represent a single combined dimension in this sample. It is possible that the factor structure is affected by the characteristics (e.g., gender, age groups, and urban/rural difference) of the population and the cultural context. The authors could not conclude from this study that the fivefactor structure fit to the Japanese PWBS-42 rather than the original six-factor model [19]. Further research is needed to re-examine the factor structure of the Japanese PWBS-42 in a sample with a broader age range and from non-urban settings to know if the pattern could be generalized to the whole Japanese population.

The results for the correlation analysis indicated that all the PWBS-42 correlated highly with other psychometrics variables measured by other scales in the theoretically expected direction, supporting its convergent validity. As shown by ANOVA, there were some significant demographic differences in scores on the Japanese version of the PWBS-42. Specifically, some differences, such as decremental age profiles (with scores for the older people significantly lower than those for younger people) for personal growth and purpose in life, sex profile (with women scoring higher than men) of positive relationship with others, were coincident with previous research in the U.S. [25]. Otherwise, high autonomy in men seems peculiar to Japanese men. Marriage had an advantage over being divorced or single in many subscales, except for autonomy. Previous studies suggested that marriage is a powerful predictor of high well-being $[53,54]$. Having paid work was related to high scores for personal growth and purpose in life. These two subscales were found to contribute to work and career related outcomes [55]. The findings supported the view that using six subscales for the Japanese population was appropriate, suggesting known-group validity compared with other population. Regarding interpretability of this scale, the result showed relatively low PWB scores among Japanese sample $(30.6<$ mean $<33.8)$ compared with the U.S. sample $(37.1<$ mean $<40.6)$. If we need to categorize individuals into low, optimal, and excessively high scores for each dimension of the PWB, such as in assessment for well-being therapy [56], this cultural difference must be considered.

\section{Limitations}

This study has some limitations. First, the MIDJA population consisted of people over 30, so young population under 29 cannot be considered. Second, the translation process is not partially appropriate. Third is that our Japanese participants treated item P7 (see Table 4) as positive even though it is negative phrased (and reversed-scored). We kept this item and its original scoring in the Japanese version of the PWBS-42 in this study to maintain consistency with the original PWBS; however, that practice should be reconsidered in future research. Fourth, some scales which were used in convergent/known-class validity analysis are biased due to insufficient consideration of reliability and validity. Finally, some important properties of the scale were not investigated in the study due to the lack of repeated measurement: test-retest reliability, measurement error, predictive validity and responsiveness.

\section{Conclusion}

The Japanese version of the PWBS-42 showed acceptable reliability and convergent validity when applying the original scoring method for the six subscales. However, the factorial validity based on the original six-factor model was not well supported in this study, while most factors observed in this study seem compatible with the original factors. A further examination is needed to know if the factor structure of the PWBS-42 is different for the Japanese population. 


\section{Supplementary information}

Supplementary information accompanies this paper at https://doi.org/10. 1186/s40359-020-00441-1.

\section{Additional file 1.}

\section{Abbreviations}

ANOVA: Analysis of variance; CFA: Confirmatory factor analysis; CFI: Comparative fit index; COSMIN: COnsensus-based Standards for the Selection of Health Measurement Instruments; EFA: Exploratory factor analysis;" MIDJA: Midlife in Japan survey; MIDUS: Midlife in the United States survey:" PANAS: The Positive and Negative Affect Schedule; PSS: The Perceived Stress Scale; PWB: Psychological Well-Being; PWBS: Psychological Well-Being Scale; RMSEA: Root mean square error of approximation; TLI: Tucker-Levis index

\section{Acknowledgments}

We appreciate the support of graduate students Mie Sudo, Yu Komase, Hiroki Asaoka, Mako lida, Mariko Suga and Risa Kotake in our efforts to collect and review the previous research on psychological well-being. Mie Sudo helped us obtain the details of data in MIDJA.

\section{Authors' contributions}

NK was in charge of this study, of supervising the process and of providing his expert opinion on the subject. NS, KW and KI organized the study design and analyzed the data. NK, MK, CK and CR conducted the national survey that comprises the data for the study. Kl, DN, MK, CK and CR ensured the accuracy or integrity of all parts of the work. All authors contributed to the process of developing and evaluating the Japanese version of the PWBS-42. NS wrote the first draft of the manuscript, and all other authors edited and revised the manuscript. All authors approved the final version of the manuscript.

\section{Funding}

This study was partly supported by MEXT KAKENHI Grant Number JP21119003 and JSPS KAKENHI Grant Number JP16H06398 both to N.K.. They do not have any role of designing the study, data collection or analysis.

\section{Availability of data and materials}

The data supporting the findings of this study are available from N.Sasaki, but restrictions apply to the availability of these data, which were used under license for the current study, and so are not publicly available. Data is available from the authors upon reasonable request and with permission from N.Kawakami.

\section{Ethics approval and consent to participate}

This study was approved by The Research Ethics Committee of the Graduate School of Medicine/Faculty of Medicine, The University of Tokyo (no. 1691(5)). Written informed consent was obtained from all participants with full disclosure and explanation of the purpose and procedures of this study. We explained that their participation was voluntary, and they could withdraw from the study at any time even after voluntarily participating without offering any reason. Participants were assured that the survey was anonymous, and no individual would be identified in analyzing and reporting the data.

\section{Consent for publication}

Not applicable.

\section{Competing interests}

None declared.

\section{Author details}

'Department of Mental Health, Graduate School of Medicine, The University of Tokyo, 7-3-1, Hongo, Bunkyo-ku, Tokyo 113-0033, Japan. ²Department of Communication, Tokyo Women's Christian University, Tokyo, Japan. ${ }^{3}$ National Institute of Occupational Safety and Health, Kiyose, Japan. ${ }^{4}$ Department of Psychology/Institute on Aging, University of Wisconsin-Madison, Madison, USA
Received: 18 February 2020 Accepted: 2 July 2020

Published online: 20 July 2020

\section{References}

1. Crumbaugh JC, Maholick LT. Manual of instructions for the purpose in life test. Munster: Psychometric Affiliates; 1969.

2. Steger MF. In: SC LSJ, editor. Meaning in life. New York: Oxford University Press; 2009 .

3. Ryan RM, Deci EL. On happiness and human potentials: a review of research on hedonic and eudaimonic well-being. Annu Rev Psychol. 2001;52:141-66.

4. Ryan RM, Deci EL. Self-determination theory and the facilitation of intrinsic motivation, social development, and well-being. Am Psychol. 2000;55(1):68.

5. Ryff CD. Beyond Ponce de Leon and life satisfaction: new directions in quest of successful ageing. Int J Behav Dev. 1989;12(1):35-55.

6. Maslow AH. Toward a psychology of being. New York: John; 1968. p. 1968.

7. Rogers CR. On becoming a person: a therapist's view of psychotherapy; 1961.

8. Ryff CD. Psychological well-being revisited: advances in the science and practice of eudaimonia. Psychother Psychosom. 2014;83(1):10-28.

9. Steptoe A, Deaton A, Stone AA. Subjective wellbeing, health, and ageing. Lancet. 2015;385(9968):640-8.

10. Kim ES, Sun JK, Park N, Peterson C. Purpose in life and reduced incidence of stroke in older adults:'The health and retirement Study'. J Psychosom Res. 2013;74(5):427-32

11. Kim ES, Sun JK, Park N, Kubzansky LD, Peterson C. Purpose in life and reduced risk of myocardial infarction among older US adults with coronary heart disease: a two-year follow-up. J Behav Med. 2013;36(2):124-33.

12. Boylan JM, Ryff CD. Psychological well-being and metabolic syndrome: findings from the midlife in the United States National Sample. Psychosom Med. 2015;77(5):548-58.

13. Mangelli L, Gribbin N, Büchi S, Allard S, Sensky T. Psychological well-being in rheumatoid arthritis: relationship to 'disease' variables and affective disturbance. Psychother Psychosom. 2002;71(2):112-6.

14. Wood AM, Joseph S. The absence of positive psychological (eudemonic) well-being as a risk factor for depression: a ten year cohort study. J Affect Disord. 2010;122(3):213-7.

15. Hamilton NA, Gallagher MW, Preacher KJ, Stevens N, Nelson CA, Karlson C, et al. Insomnia and well-being. J Consult Clin Psychol. 2007;75(6):939-46.

16. Boyle PA, Buchman AS, Barnes LL, Bennett DA. Effect of a purpose in life on risk of incident Alzheimer disease and mild cognitive impairment in community-dwelling older persons. Arch Gen Psychiatry. 2010;67(3):304-10.

17. Morozink JA, Friedman EM, Coe CL, Ryff CD. Socioeconomic and psychosocial predictors of interleukin-6 in the MIDUS national sample. Health Psychol. 2010;29(6):626.

18. McKinley NM. The developmental and cultural contexts of objectified body consciousness: a longitudinal analysis of two cohorts of women. Dev Psychol. 2006;42(4):679.

19. Ryff CD. Happiness is everything, or is it? Explorations on the meaning of psychological well-being. J Pers Soc Psychol. 1989;57(6):1069.

20. Ryff CD. Well-being with soul: science in pursuit of human potential. Perspect Psychol Sci. 2018;13(2):242-8.

21. Cheng S-T, Chan ACM. Measuring psychological well-being in the Chinese. Pers Individ Dif. 2005:38(6):1307-16.

22. Iwano $\mathrm{S}$, et al. Development of a brief Psychological Well-Being Scale. Behav Sci Res. 2018:54(1):9-21 (Japanese).

23. Nishida Y. Adult women's lifestyle and psychological well-being. Educ Psychol Stud. 2000;48(4):433-43. (Japanese) doi. https://doi.org/10.5926/ jjep1953.48.4_433:433-43.

24. Kishida Y, Kitamura T, Gatayama R, Matsuoka T, Miura S, Yamabe K. Ryff's psychological well-being inventory: factorial structure and life history correlates among Japanese university students. Psychol Rep. 2004;94(1):83-103.

25. Ryff $C D$, Keyes $C L$. The structure of psychological well-being revisited. J Pers Soc Psychol. 1995:69(4):719-27.

26. Ryff CD, Lee $\mathrm{YH}$, Essex MJ, Schmutte PS. My children and me: midlife evaluations of grown children and of self. Psychol Aging. 1994;9(2):195.

27. Schmutte PS, Ryff CD. Personality and well-being: reexamining methods and meanings. J Pers Soc Psychol. 1997;73(3):549.

28. Research I-uCfPaS. Midlife in the United States (MIDUS 2), 2004-2006 Documentation of Psychosocial Constructs and Composite Variables in MIDUS II Project 1 [Available from: https://www.icpsr.umich.edu/icpsrweb/ ICPSR/studies/4652. 
29. Ryff CD KS, Karasawa M, Markus H, Kawakami N, Coe C. Survey of Midlife Development in Japan (MIDJA), 2008 [Computer file]. ICPSR30822-v2 2008

30. Mokkink LB, de Vet HCW, Prinsen CAC, Patrick DL, Alonso J, Bouter LM, et al. COSMIN risk of Bias checklist for systematic reviews of patient-reported outcome measures. Qual Life Res. 2018;27(5):1171-9.

31. Wild D, Grove A, Martin M, Eremenco S, McElroy S, Verjee-Lorenz A, et al. Principles of good practice for the translation and cultural adaptation process for patient-reported outcomes (PRO) measures: report of the ISPOR task force for translation and cultural adaptation. Value Health. 2005:8(2):94-104.

32. Research I-UCfPaS. Surveyof Midlife in Japan (MIDJA), April-September 2008 (ICPSR 30822) -Documentation of Scales and Constructed Variables in MIDJA.

33. Watson D, Clark LA, Tellegen A. Development and validation of brief measures of positive and negative affect: the PANAS scales. J Pers Soc Psychol. 1988;54(6):1063-70.

34. Clobert M, Sims TL, Yoo J, Miyamoto Y, Markus HR, Karasawa M, et al. Feeling excited or taking a bath: Do distinct pathways underlie the positive affect-health link in the U.S. and Japan? Emotion. 2019;20(2):164-78.

35. Mroczek DK, Kolarz CM. The effect of age on positive and negative affect: a developmental perspective on happiness. J Pers Soc Psychol. 1998;75(5): 1333-49.

36. Rosenberg M. Society and the adolescent self-image: Princeton university press; 2015.

37. Kan C, Kawakami N, Karasawa M, Love GD, Coe CL, Miyamoto Y, et al. Psychological resources as mediators of the association between social class and health: comparative findings from Japan and the USA. Int J Behav Med. 2014;21(1):53-65.

38. Cohen S. Perceived stress in a probability sample of the United States; 1988.

39. Cohen S, Kamarck T, Mermelstein R. A global measure of perceived stress. J Health Soc Behav. 1983;24(4):385-96.

40. Guttman L. Some necessary conditions for common-factor analysis. Psychometrika. 1954;19(2):149-61.

41. Kaiser HF. The application of electronic computers to factor analysis. Educ Psychol Meas. 1960;20(1):141-51.

42. Kaiser HF. A second generation little jiffy. Psychometrika. 1970;35(4):401-15.

43. Keyes CLM, Shmotkin D, Ryff CD. Optimizing well-being: the empirical encounter of two traditions. J Pers Soc Psychol. 2002;82(6):1007-22.

44. Clarke PJ, Marshall WW, Ryff CD, Wheaton B. Measuring psychological wellbeing in the Canadian study of health and aging. Int Psychogeriatr. 2001; 13(S1):79-90.

45. Markus HR, Kitayama S. Culture and the self: implications for cognition, emotion, and motivation. Psychol Rev. 1991;98(2):224.

46. Kan C, Karasawa M, Kitayama S. Minimalist in style: self, identity, and wellbeing in Japan. Self Identity. 2009:8(2-3):300-17.

47. Abbott RA, Ploubidis GB, Huppert FA, Kuh D, Wadsworth ME, Croudace TJ. Psychometric evaluation and predictive validity of Ryff's psychological wellbeing items in a UK birth cohort sample of women. Health Qual Life Outcomes. 2006;4(1):76.

48. Ryff $C D$, Singer $B H$. Best news yet on the six-factor model of well-being. Soc Sci Res. 2006;35(4):1103-19.

49. Watanabe $\mathrm{K}$, et al. Measuring eudemonic well-being at work: a validation study for the 24-item The University of Tokyo Occupational Mental Health well-being scale among Japanese workers. Ind Health. 2020;58(2).

50. Riazi A, Bradley C, Barendse S, Ishii H. Development of the well-being questionnaire short-form in Japanese: the W-BQ12. Health Qual Life Outcomes. 2006;4:40

51. Iwata N, et al. Job stressor-mental health associations in sample of Japanese working adults: artifacts of positive and negative questions? Ind Health. 1999;37(2):263-70.

52. Iwata N, Roberts CR, Kawakami N. Japan-US comparison of responses to depression scale items among adult workers. Psychiatry Res. 1995;58(3):237-45.

53. Wilson CM, Oswald AJ. How does marriage affect physical and psychological health? A survey of the longitudinal evidence; 2005.

54. Gove WR, Hughes M, Style CB. Does Marriage Have Positive Effects on the Psychological Well-Being of the Individual? J Health Soc Behav. 1983;24(2): 122-31.

55. Strauser DR, Lustig DC, Çiftçi A. Psychological well-being: its relation to work personality, vocational identity, and career thoughts. J Psychol. 2008;142(1):21-35.

56. Fava GA. Well-being therapy: conceptual and technical issues. Psychother Psychosom. 1999;68(4):171-9.

\section{Publisher's Note}

Springer Nature remains neutral with regard to jurisdictional claims in published maps and institutional affiliations.
Ready to submit your research? Choose BMC and benefit from:

- fast, convenient online submission

- thorough peer review by experienced researchers in your field

- rapid publication on acceptance

- support for research data, including large and complex data types

- gold Open Access which fosters wider collaboration and increased citations

- maximum visibility for your research: over $100 \mathrm{M}$ website views per year

At $\mathrm{BMC}$, research is always in progress.

Learn more biomedcentral.com/submissions 